\title{
Tracks in the Snow
}

\section{Catherine Xu, Department of English, Indiana University - Bloomington}

Faculty Mentor: Dr. Christoph Irmscher

\section{ABSTRACT}

In 2000, Subhankar Banerjee set out for the Arctic National Wildlife Refuge to photograph polar bears in a "place untrammeled by tourism or industry" (Banerjee, 2008). This paper explores a number of threads regarding Banerjee's artistic journey from descriptive to interpretive work, including the role of politics in Banerjee's evolution as an artist and environmental activist and comparisons of his different publications over time. Along with providing context for Banerjee's work, this paper investigates the unique avenues through which Banerjee's photography challenges the traditional paradigm of a pristine wilderness by reconceiving its spacial representations in exhibitions and books highlighting the presence of humans in the Arctic. Research was primarily conducted through an analysis of both photographic and textual elements of Banerjee's publications and a conversation with Banerjee.

KEYWORDS: environmental photography, art activism, wilderness paradigm, environmentalism

\section{INTRODUCTION}

The sun beams on the yellow-white fur of a polar bear as 1 it lumbers towards the leftover bones of a whale, mouth slightly open in anticipation of its meal (see Figure 1). Between patches of half-melting ice, water reflects the bright blue of the sky and a perfect mirror image of the bear. You might recognize this photograph-it has been wielded by scores of environmentalists, politicians, and journalists decrying global warming. Ironically, its photographer, Subhankar Banerjee (b. 1967), did not have political intentions when he first set out to photograph the Arctic; back then, his main motivation was to seek inspiration in "a place untrammeled by tourism or industry, a place untouched by man, pristine wilderness, so called 'the last frontier"' (Banerjee, 2008). However, this view of the Arctic would come to change as Banerjee immersed himself in Arctic life, producing art that, both intrinsically and through its special presentations, conveys a narrative of ecological richness that deconstructs the wilderness paradigm of classic American conservationism. In addition to his venture north, Banerjee underwent a political journey, transforming the way he would view and approach art as a vehicle for change.

\section{SEASONS OF LIFE AND LAND}

Before becoming a professional photographer, Banerjee was an engineer for Boeing and backpacking enthusiast, and before that, he was a boy growing up in Calcutta, drawn to images in the form of painting and the cinema. Coming from a middle-income family, however, he decided to take the "practical" route and pursue a career in the sciences. After his graduate studies brought him to New Mexico State University, Banerjee fell in love with the wide-open spaces of the American southwest. He joined the Sierra Club, where he became interested in the restoration of natural spaces, and would often go backpacking, taking photographs along the way. It was in part this passion for photographing the wild that led him to take his career to Seattle, where he

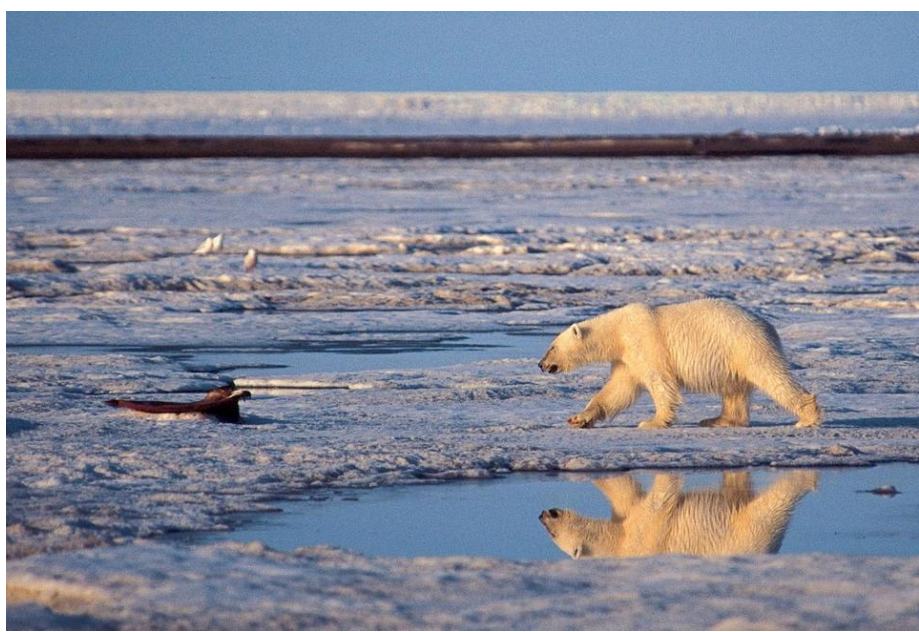

Figure 1. Banerjee, Polar Bear on Bernard Harbor (2001). Reproduced with permission by S. Banerjee.

joined a mountaineering club. In 2000, Banerjee ventured to Manitoba, a popular destination for people seeking to photograph polar bears, but was dissatisfied with his experience of commercialized tourism and industry. Craving a more authentic exposure to the wilderness, Banerjee left his job at Boeing and headed for the Arctic National Wildlife Refuge (Banerjee, 2012b).

Once in the Arctic, Banerjee's romantic notions of a journey through the wilderness were quickly dispelled by weather that was so brutal his camera lens shattered from the cold. "There were times I was sure I would freeze to death until another cup of hot coffee revived me, times when there was no money and only the richness of fellowship could buy us sufficient hope for another day's work," he recalled (Banerjee, 2003, p. 17).

Yet he also found the land to be beautiful and nurturing, bursting with rich, ecological vitality four seasons a year. Banerjee's first book, Arctic National Wildlife Refuge: Seasons of Life and Land (2003), reflects this realization, bringing the Arctic back to the traveler at home. Filled with 
14 months worth of evocative photographs, entries from his diary, and essays from prominent environmentalist writers, the book journalistically recounts Banerjee's first Arctic experience.

One of the most artistically powerful aspects of this first book, however, lies in its presentation of wildlife photography in clusters, implying a temporal continuity that reminds the viewer that the Arctic is a living place. For example, two photographs displayed one above the other show a moose feeding on vegetation. The top photograph shows the moose with its head raised, eating from an upper branch. The photograph directly underneath is nearly identical, but the moose has lowered its neck and is now feeding on a lower branch (Banerjee, 2003, p. 20). By evoking a sense of motion, this simple difference-a lowered neck-transcends the frozen stillness of photography. The Arctic, to the viewer, is no longer a two-dimensional slice of Banerjee's journey; it is a land that exists in time and space, constantly moving and changing-a land with which one can actually interact.

\section{ROLE OF SPATIAL PRESENTATION}

Many of Banerjee's later images also reinforce an ecological narrative through the way they are presented in Banerjee's publications, often conveying the interconnectedness of the Arctic life and land. The aerial photographs in Banerjee's book Resource Wars (2008) do this especially well in two predominant styles. For the first, Banerjee sequentially presents two images that are identical except for a change in scale. For example, a photograph titled "Caribou Crossing the Utukok River” (Banerjee, 2008, p. 18) depicts a vast landscape in which green vegetation takes up the majority of the page and patches of snow delineate a silt-grey river bend. To the right is the same image, but expanded to three pages and magnified so that one can almost see the individual parts of the caribou. It's as if the first image says, "Here is an Arctic landscape," while the second demands, "Look closer. Here is a living ecosystem." The combination of the two is a powerful reminder not to dismiss the landscape as only an expanse of land; it is also a habitat filled with life and motion.

Less commonly, Banerjee will reveal a closer shot of a view first and then zoom out in the following page, such as with "Brant and Snow Geese with Chicks" (Banerjee, 2008, p. 41). By showing the geese on a more individual level first and then in a broader scope, Banerjee seems to comment that one must not see them as restricted to the area in view. In other words, Banerjee does not let his audience get away with viewing one part of nature without the whole context. There are the geese, yes, but then there is the land, and to look at the geese, one must look at the land as well.

The second ecologically evocative layout Banerjee employs in Resource Wars is the placement of two images side by side that are distinct but similar in color and texture. A two-page spread containing "Sheenjek River II: A Gwich'in Homeland" (Banerjee, 2008, p. 66) and "Caribou Migration II" (Banerjee, 2008, p. 67) demonstrates this technique. Both images depict vast landscapes of white snow and blue ice, and a mountain line from the former seems to continue smoothly into the latter. The long lines of caribou treading across the ice in the latter image do not explicitly pass through the former, but the combination of the two creates a sense of the extensive distance the caribou must travel. The two pictures seem to be extensions of each other, reminding the audience that the landscape exists beyond the end of the page.

\section{PEOPLE OF THE LAND AND THE WILDERNESS PARADIGM}

The first photograph in that same spread also makes another more indirect, but perhaps even more important point; to notice it, the viewer must look not to the image itself, but its caption, "Sheenjek River II: A Gwich'in Homeland." Paradoxically, the image itself contains no visible signs of human presence; yet that is precisely what makes it so important. Taken at face value, the landscape shown reinforces the pre-existing beliefs of many American conservationists: that the Arctic is an empty and pristine wilderness, an untouched land. The title, however, points out what the viewer missed by just gazing at the surface. Despite what the viewer thinks or how nature is presented, the Arctic is the homeland to a group of people and the viewer must learn to reframe it as such.

In the foreword of Banerjee's collection Arctic Voices (2012), the artist recounts an instance in which, after viewing Banerjee's work, a young environmentalist asked, "How could there be a hunting camp in a pristine wilderness?" (Banerjee, 2012, p. 15). The environmentalist's confusion, while not ill-intended, is indicative of a dangerous and prevalent mentality in classic American conservationism, which is based largely on the idea that wilderness and humans are completely separate. This notion is not only idealistic and false, but also severely detrimental. During the conception of Yellowstone and Yosemite National Parks, conservationists would completely ignore the clear presence

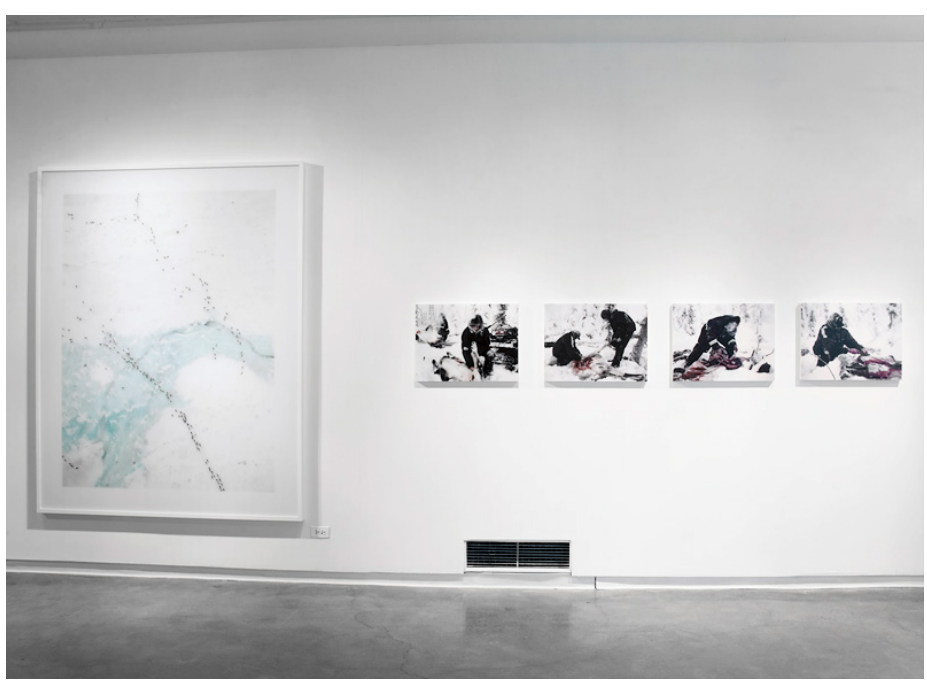

Figure 2. Banerjee, Installation View (2009). Reproduced with permission by $\mathrm{S}$. Banerjee. 
of at least five tribes in the area, reporting lands they deemed conservation worthy as empty, untrodden, and pristine. Native Americans and rural, white subsistence-hunters would be labeled "thieves," "poachers," and "squatters," and would forcibly be moved from their own homelands to preserve the conservationists' ideal. For 32 years, the U.S. military ran Yellowstone, effectively enforcing cultural eradication (Banerjee, 2012a, p. 9).

While Banerjee may have embarked for the Arctic with the ideal of a pristine landscape in mind, his interactions with the Gwich'in and Inupiat people ran completely counter to the classic wilderness paradigm. Banerjee's guide on his first trip to the wilderness was an Inupiat hunter named Robert Thompson, who, along with his wife Jane, taught Banerjee how to survive in the Arctic. In an immersive experience, Banerjee learned about Inupiat culture and documented the sacred whale hunt. He also lived with the Gwich'in people for a time, going on caribou hunts and learning the importance of the caribou to Gwich'in culture and life. Consequently, Banerjee's work in the Arctic breaks the wilderness paradigm not only by including native Inupiat and Gwich'in people in his photography, but by depicting them as having an established place in the ecology of the Arctic and a culture tethered to the land.

One such example of human-Arctic interrelations comes from a group of Banerjee's photographs in an exhibit (2009) in Jaffe-Friede Gallery in the Hopkins Center for the Arts, which elevates the narrative of humans as a part of Arctic ecology by simply having the images share a wall (see Figure 2). On the left is an $86 \times 68$ " aerial shot of long lines of migrating caribou taken at such a distance that the caribou look no larger than ants, the white ice beneath them divided by a spread of blue. The image is juxtaposed to the right with a significantly smaller five photo series of Gwich'in hunters butchering the very same species of animal seen trekking over ice on the left (Banerjee, 2009). Blooddark in the snow, there is no glorification of the hunter or hunted; these photographs are close and confrontational, and, compounded with the caribou migration image, force the audience to recognize both the reality of nature and the essential connection between land, animal, and human. This point is bolstered by the fact that, in the two images, the smears of red caribou blood and flesh and the blue of the ice supporting the caribou from underneath are the only colors present. The blood of the caribou (its death and flesh) is necessary for the survival of the Gwich'in people, and the ice (the land of the Arctic coast) is necessary for the survival of the caribou.

Banerjee's famous photograph of a polar bear lumbering across melting ice towards whale bones (see Figure 1) also exemplifies the interdependence of people and Arctic wildlife. The caption tells what the photograph on its own cannot: that the whale bones were leftover by the Inupiat from the previous year's hunt; the polar bear and other scavenging animals now benefit from the presence of humans.

A more solemn image highlighting human-Arctic involvement shows an Inupiat cemetery. Two massive jawbones of a bowhead whale arch out of the snow and into the sky in the form of a cemetery gateway, illustrating how the relationship the Inupiat people have with the bowhead whales is so deep that they are together even in their final resting places. Towards the back of the image, Christian crosses made of whalebone mark each grave. Together, the familiar (though perhaps unexpected) Western and unfamiliar tribal symbols evoke empathy in the viewer. Despite the solemnity of the image, the cemetery is not an inherently sad place; the sadness arises from the scene of past generations of Inupiat people buried in their land, the very land that the wilderness paradigm of Western civilization denies has even been trodden on by human feet. This denial is worse than ignorance; it is cruel cultural erasure.

\section{THE TIPPING POINT}

In an even graver depiction of human involvement in the Arctic, an exposed coffin by the shore takes the foreground (see Figure 3). The coffin, originally buried in permafrost, now only has a thin layer of dirt to cover it (Banerjee, 2006). Unlike the photograph of the Inupiat cemetery, this image points less to the people who buried the coffin than to the people thousands of miles away who are unknowingly responsible for exhuming it. Banerjee took this photograph in 2006, six years after his initial trip to the Arctic. In the span of those years, so much had changed so quickly. Banerjee saw scenes of the Arctic he had never seen before: "a drunken forest in Siberia, trees leaning at odd angles from softening of the permafrost; and the skeleton of caribou that had died from starvation due to winter icing on the tundra" (Banerjee, 2012a, p. 2). In addition, whereas there was little talk about global warming in the Arctic communities the first time he visited, though oil development was frequently discussed, climate change was on everyone's tongue five, six, or seven years later-how it was affecting the animals, communities, and Arctic ecology.

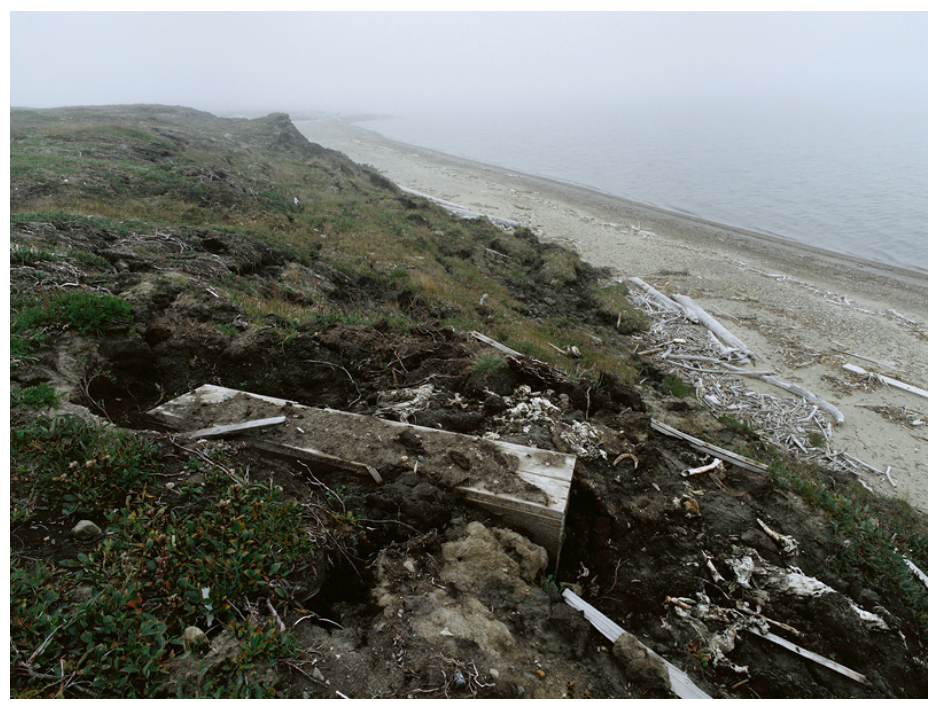

Figure 3. Banerjee, Exposed Coffin (2006). Reproduced with permission by $\mathrm{S}$. Banerjee. 
While Banerjee was noticing changes in the Arctic firsthand, a political war was raging back in Washington D.C. regarding whether or not companies should have the right to drill in the Arctic National Wildlife Refuge. Prominent Republicans and the Bush administration certainly thought so; the Secretary of the Interior at the time, Gail Norton, characterized the Arctic as lifeless, a "flat, white, nothingness." One Republican senator from Alaska held up a blank poster board and said, "This is a picture of Arctic National Wildlife Refuge as it exists for about nine months of the year." In March 2003, Senator Barbara Boxer of California challenged that view of the Arctic. "Cast your eyes on this," she said on the Senate floor, displaying the very image of a polar bear lumbering across ice mentioned earlier along with many of Banerjee's other photographs from Seasons of Life and Land. Her amendment that would prevent (or at least delay) drilling in the Arctic National Wildlife Refuge passed, 52-48 (Dunaway, 2009).

This use of Banerjee's work in a high-profile political setting catalyzed a turning point in his career. Banerjee had originally intended for his work to be educational and stayed mostly away from political focus groups. After Senator Boxer's use of his photography, however, Banerjee realized the impact his work could have and how photography could be used as a vehicle for change (S. Banerjee, personal communication, December 8, 2015). Much more so than for other art forms, people believe what nature photography shows them. Every image implies reality; the photographer must have been a firsthand witness of the scene-touched by the light, nose in the flowers, cheeks stung from the cold-to have brought the experience back for viewers to share.

As Banerjee's aims turned political, many aspects of his work began to change. While his earlier photography aimed to show a descriptive picture of the Arctic, his focus shifted to a more interpretive view, polished and pointed like a weapon. In the first sentence of Resource Wars, Kelley Wilder proclaims that aerial photography, the style Banerjee predominantly employs in the book, "is the language of war" (Banerjee, 2008, p. 11). Indeed, looking through Seasons of Life and Land, viewers mostly see the Arctic with their feet on the ground, as they would were they actually there. By contrast, viewers see the striking aerial landscapes in Resource Wars from the sky, page after page after page. Banerjee's photographs acknowledge the physical space that separates the viewer from the landscape. Yet they also compel them to understand how connected they are to the land. The difference is clear: the first style focuses on the individual; the second, by showing the literal big picture, demands that the viewer recognize the figurative big picture of global connectivity and how our decisions at home have a real effect on places far away.

\section{SHIFT TO WRITING}

In 2007, Banerjee began drawing back from pure photography, instead placing a greater emphasis on writing, both in essays and as an accompaniment to his art (S.
Banerjee, personal communication, December 8, 2015). This transition is reflected in the different ways in which Banerjee describes the same pieces of his own work in Seasons of Life and Land (2003), and then again in Arctic Voices (2012a). In the former, four photographs depict a polar bear mother and her two cubs as they tread through the snow then rest, the mother stationary while the cubs bound around her, each vying for her attention (Banerjee, 2003, p. 24). The image is sympathetic, and the two-line caption informs the reader about the general timeline of polar bear motherhood. When Banerjee writes again about these photographs, in his collection Arctic Voices (2012), his tone is drastically different:

This isn't an adventure story, as you might think, but instead, as you'll see later in this volume, we ask such questions as, "Can you imagine the oil companies cleaning up a BP-like spill in the frozen Arctic Ocean in such a blizzard? (Banerjee, 2012a, p. 6)

This new commentary effectively shifts the topic of conversation away from being contained within the Arctic, instead pointing to a specific political issue and even naming an associated corporation that readers will undoubtedly recognize.

A 2001 photograph of musk oxen serves as another example of this change in intention (Banerjee, 2012a, p. 1). Located in one of the first few pages of Seasons of Life and Land, the oxen are silhouetted against a completely orange sky, the tops of their backs appearing almost to dissolve into the orange fog. Beneath them, the snow is such a deep blue that it could almost pass for the sea. In this book, the image is caption-less, but in Arctic Voices (2012), Banerjee provides sharp commentary:

I began to wonder how could there be such vibrant colors in an environment that is supposed to be free of pollution? I remember from my childhood many colorful sunrises and sunsets in Kolkata, where pollution in the air was all around us; it still is. There had to be particulates in the air to create those deep redorange colors in the musk oxen photo. (Banerjee, 2012a, p. 8)

Again, Banerjee now chooses to focus less on the subject than on the environmentalist implications of the image.

Gone was Banerjee's previous, more journalistic treatment of the Arctic. Each photograph in his later collections is imbued with purpose, bolstered by pointed textual commentary that explicitly and specifically conveys the urgency of environmentalism. In Resource Wars (2008), with a leaflet tucked into the front cover that serves as a complement to the images, Banerjee informs the reader not only about the detrimental effects of climate change on the native population and entire Arctic ecosystem, but he also makes sure that it is very clear that the fight is right at the reader's doorstep, instilling them with responsibilitycompelling them to recognize their own personal stake in what happens in the Arctic.

\section{CONCLUSION}

While the politics in many of Banerjee's works directly involve the Arctic, an underlying theme of global connectivity 
is always present. After all, if this far-away place often conceptualized as the last pristine wilderness is so affected by the decisions we make at home, how can we consider ourselves and our actions isolated from the rest of the world? It is not enough to prevent industrialization in the Arctic; our choices at home matter as well. Arctic ecology is world ecology. It affects the ecology of the spaces we choose to inhabit and we are constantly and personally engaging with all of it. "It may be just a square inch of space," Banerjee told me, "but square inches make up cities" (S. Banerjee, personal communication, December 8, 2015).

Recently appointed a Cambridge Fellow, Banerjee has spent a great deal of time reconsidering the concept of wilderness. When I asked him what wilderness means to him now, he replied, "sustenance: spiritually, culturally, and nutritionally" (S. Banerjee, personal communication, December 8, 2015). This new view of the wilderness places an emphasis on inclusion-of the feeling that originally drew him and other conservationists to nature, of the people who call it their homeland, and of all the living things that make up its vibrant ecology. Banerjee's work is an attempt at reconciliation, acknowledging the history of the outdated wilderness paradigm while bringing back the unfiltered voices of the Arctic.

If we now return to what has perhaps become Banerjee's most famous photograph, we see, once again, the sun beaming on the yellow-white fur of a polar bear lumbering towards its meal, left over from an Inupiat hunt. We see, between patches of half-melting ice, a crisp reflection of the bear and the bright sky. But now we see it differently. Banerjee might have gone to the Arctic to capture precisely this moment, but we know he gained so much more-the impetus to work towards change in the world.

\section{AUTHOR INFORMATION}

All correspondence should be sent to xucat@iu.edu.

\section{ACKNOWLEDGMENTS}

The author would like to thank Christoph Irmscher for his support and feedback throughout the process of writing this manuscript. Additional thanks are extended to Subhankar Banerjee for his kindness in taking the time for an interview.

All images are reproduced with the permission of the photographer (S. Banerjee) and may not be reproduced or distributed without consent of the photographer.

Figures 1-3 (C) 2001-2009 Subhankar Banerjee.

\section{REFERENCES}

About Subhankar Banerjee. (2015). Subhankar Banarjee.

Retrieved from: http://www.subhankarbanerjee.org/

banerjee.html
Banerjee, S. (Photographer). (2001). Polar bear on Bernard Harbor [photograph]. Retrieved from: https:// www.bostonglobe.com/news/nation/2015/11/25/ climate-change-driving-more-polar-bears-onto-land/ iOQWuNPYPxC1ow2ofa6GOM/story.html

Banerjee, S. (Photographer). (2006). Exposed coffin [photograph]. Retrieved from http://www. subhankarbanerjee.org/photohtml/arctic-photo-greengrey-09.html

Banerjee, S. (Photographer). (2009). Installation view [photograph]. Retrieved from http://www. subhankarbanerjee.org/photohtml/arctic-photowhite-05.html

Banerjee, S. (2003). Seasons of life and land: Artic National Wildlife Refuge. Seatle, WA: The Mountaineers Books.

Banerjee, S. (2008). Resource wars. New York, NY: Sundaram Tagore Gallery.

Banerjee, S. (2012a). Arctic voices: Resistance at the tipping point. New York, NY: Seven Stories Press.

Banerjee, S. (2012b). "Once a physicist: Subhankar Banerjee [Interview].” Retrieved from: http://www.iop. org/careers/workinglife/profiles/page_37729.html

Dunaway, F. (2009). Reframing the Last Frontier. In A.C. Braddock \& C. Irmscher (Eds.), A keener perception: Ecocritical studies in American art history (pp. 254274). Tuscaloosa, AL: University of Alabama Press. 